\title{
Asymptotic theory for a moving droplet driven by a wettability gradient
}

\author{
Len M. Pismen* \\ Department of Chemical Engineering and Minerva \\ Center for Nonlinear Physics of Complex Systems, \\ Technion - Israel Institute of Technology, Haifa 32000, Israel \\ Uwe Thielet \\ Max-Planck-Institut für Physik komplexer Systeme, \\ Nöthnitzer Str. 38, D-01187 Dresden, Germany
}

\begin{abstract}
An asymptotic theory is developed for a moving drop driven by a wettability gradient. We distinguish the mesoscale where an exact solution is known for the properly simplified problem. This solution is matched at both - the advancing and the receding side - to respective solutions of the problem on the microscale. On the microscale the velocity of movement is used as the small parameter of an asymptotic expansion. Matching gives the droplet shape, velocity of movement as a function of the imposed wettability gradient and droplet volume.
\end{abstract}




\section{INTRODUCTION}

The description of the movement of a three-phase contact line is an essentially unsolved hydrodynamical problem that continues to attract much interest, for instance, when studying spreading drops, and liquid sheets or ridges moving down an inclined plate. The understanding of 'simple' contact line movement is also paramount for a deeper insight in related problems as the dynamical wetting transition and transversal instabilities of moving contact lines.

It is well known that the divergent shear stress at the contact line forbids a solution in the framework of purely classical hydrodynamics, i.e. assuming a no-slip boundary condition at the solid-liquid interface. Although this was first pointed out by Huh and Scriven ${ }^{1}$ based on Moffatt' ${ }^{2}$ solution for flow in the edge which does not satisfy the normal stress boundary condition on a free interface, and was never proven rigorously, the divergence can be understood as a consequence

of incompatibility of multivaluedness of the velocity at the contact point in the classical hydrodynamic formulation.

The boundary condition has to be relaxed to permit movement of the contact line. This can be done by introducing a very thin precursor film on the 'dry' substrate ${ }^{3}$, or by allowing for slip at the solid-liquid interface everywhere ${ }^{\frac{1}{-}}$ or only near the contact line $e^{4.5}$, or introducing an effective molecular interaction between the substrate and liquid into the hydrodynamic model ${ }^{6.7}$. For a discussion of the slip condition see also the review by Dussan ${ }^{8}$. Other approaches include phase changes at the contact line ${ }^{\frac{9}{2}}$ or introduce the vapour-liquid or fluid-solid interface, or both, as separate phases with properties that differ from the bulk fluid ${ }^{10}$.

Most of the work on moving liquid sheets and ridges prescribes a precursor film or slip at the substrate. Divergence problems at the contact line are avoided, but at the expense of introducing ad hoc parameters into the theory. These, namely the slip length or the precursor film thickness, influence the profile of ridges and fronts and hence also the characteristics of the transverse instability ${ }^{3 \cdot 11.12 .13}$.

The most realistic option is the explicit introduction of molecular interactions into the hydrodynamic formalism. This is accomplished by means of an additional pressure term, the disjoining pressure ${ }^{14}$. Depending on the particular problem treated, this disjoining pressure may incorporate long-range van der Waals and/or various types of short-range interaction terms ${ }^{6,7.15}$. Recently Pismen $^{16}$ derived a film thickness equation with a disjoining pressure term by combining the long wave approximation for thin films ${ }^{17}$ with a nonlocal diffuse interface description for the liquid-gas 
interface that incorporates van der Waals interactions.

These interactions are essential for the process of dewetting, and studies of dewetting of a thin liquid film on a substrate are generally based on models involving a disjoining pressure $18,19,20,21,22,23$. Only a few studies of instabilities of liquid fronts have adopted a similar approach $^{24,25,26}$, despite the fact that such an approach predicts all the ad hoc parameters of the slip or precursor models (i.e., the static and dynamic contact angle, drop velocity, and the drop and precursor film thickness) connected with the wetting properties of the liquid in terms of the parameters characterizing the disjoining pressure.

Recently, Eggers presented asymptotic solutions for the profile of advancing ${ }^{27}$ and receding ${ }^{28}$ driven contact lines (see also Ref.29). The respective solutions match inner solutions near the contact line where a slip model is used and outer solutions based on an analytic solution in terms of Airy functions discussed in Refs. 30.31.32.33. However, the advancing and receding case are studied for a plate pushed into and pulled out of a liquid bath, respectively. It is not possible to directly couple the two asymptotic solutions to describe the motion of a driven moving droplet or ridge.

In the present work we tackle the problem of an asymptotic description of a gradient-driven moving droplet that encompasses both an advancing and a receding contact line. This implies that the description of the two contact lines and the respective matching procedures depend on each other. Thereby we explicitly introduce the molecular interactions into the hydrodynamic formalism by using a chemical potential or disjoining pressure describing a situation of partial wetting. This corresponds to a precursor film model where the precursor film thickness is determined through the disjoining pressure.

We distinguish among three regions:

- Microscopic (molecular scale) region: the dominant balance is between disjoining potential and surface tension.

- Mesoscopic region: the dominant balance is between viscous dissipation and surface tension.

- Macroscopic region: the dominant balance is between surface tension and external forces Examples for driving forces are gravity for droplets or fronts on inclined plates, Marangoni forces occurring if temperature gradients along the substrate exist or wettability gradients along the substrate. Both, gravity and Marangoni forces act in the lubrication limit as bulk forces, i.e. the force 
is fed into the system in a top-down manner. This implies that the macroscopic region has to be included in the description. However, the third mentioned way to drive the system is based on a force resulting from a wettability gradient that is fed into the system in a bottom-up manner, i.e. on the microscale. The simplest description of such a system is undertaken here by matching solutions obtained in the mesoscopic and microscopic region. If the droplets are small enough (smaller than the capillary length) the macroscopic scale can be ignored.

There are different physical situations where a gradient in wettability occurs that can be mapped onto the presently studied model. (i) A droplet can 'sit' on a step in wettability ${ }^{34}$ allowing for an intermittent range of stationary movement until the complete drop sits on the more wettable substrate. (ii) A droplet can move along a smooth wettability gradient 35.36 .37 .38 . (iii) In a situation involving an adsorption reaction at the substrate underneath the droplet a droplet can produce the wettability gradient that drives its movement ${ }^{39,40,41,42}$. In this way it carries the gradient along with its movement. The latter case is also related to droplet motion caused by a surface phase transitions $\stackrel{43}{ }$.

In the following we study all these situations in a model that uses a chemical potential with different constants at the advancing and the receding contact line, respectively. For situations (ii) and (iii) this corresponds to the assumption that the wettability gradient is small as compared to the size of the contact zone but sizable as compared to the overall droplet size.

In the next section the basic equations for the lubrication description of moving droplets are introduced. The exact solution in the mesoscopic region and its asymptotics are described in Section III The microscopic solution and its asymptotic matching are discussed in Section IV Finally, a comparison of asymptotic and numerical results is given together with our conclusions in section $\mathrm{V}$

\section{BASIC EQUATIONS}

Our starting point is the thin film evolution equation in lubrication approximation

$$
\partial_{t} h=-\nabla \cdot\left\{k(h) \nabla\left[\gamma \epsilon^{2} \nabla^{2} h-\widehat{\mu}_{s}(h)\right]\right\}
$$

Here $\gamma$ is the surface tension of the liquid; $\epsilon$ is a scale ratio used as a small parameter of the lubrication expansion (which will further be identified with the local equilibrium contact angle). We shall use the simplest mobility function $k(h)=\eta^{-1} h^{3} / 3$, obtained under assumption of constant 
dynamic viscosity $\eta$ with no slip at the substrate. The chemical potential $\widehat{\mu}_{s}$ accounts for wetting properties. Note, that it corresponds to the negative of a disjoining pressure $\Pi$ as used, for instance, in Ref.44. For specific computations, we shall use the form ${ }^{16}$

$$
\widehat{\mu}_{s}(h)=\frac{Q_{s}}{h^{3}}\left[1-\left(\frac{h_{m}}{h}\right)^{3}\right],
$$

where $Q_{s}$ is a characteristic excess fluid-substrate interaction energy, which is proportional to the Hamaker constant ${ }^{15}$. If $Q_{s}>0$, this form corresponds to a negative long-range and positive short-range part of the spreading coefficient, thereby combining a destabilizing long-range and a stabilizing short-range van der Waals interaction. The contact angle is finite, and bulk fluid coexists at $\Pi=0$, i.e. in a flat layer of macroscopic thickness in the absence of external forces, with an ultrathin precursor of thickness $h_{m}$.

The variables in Eq. (1) are still dimensional but scaled to conform with the lubrication approximation. They are related to the physical variables (marked by a hat) as follows:

$$
\widehat{h}=h, \quad \widehat{x}=x / \epsilon, \quad \widehat{t}=t / \epsilon^{2} .
$$

In consequence the scaled contact angle $\theta$ is related to the physical one by $\theta=\widehat{\theta} / \epsilon$; the scaled droplet volume $V=\int h d x$ is related to the physical one $\widehat{V}=\int \widehat{h} d \widehat{x}$ by $V=\epsilon \widehat{V}$. Without any gradient parallel to the substrate, this model describes droplets with a finite equilibrium contact angle sitting on an ultrathin precursor film.

However, here we are interested in moving droplets driven by wettability gradients along the substrate. In the chemical potential chosen here [Eq. (2)] a wettability increase can be modelled by a decrease of $Q_{s}$ or by an increase of $h_{m}$. We chose here the former possibility. Note, however, that in a real physical system both parameters are affected. The analysis then involves more algebra but is also straightforward.

We shall consider stationary motion of a 2D droplet with the velocity $U$. Replacing in Eq. (1) $\partial_{t} h$ by $-U h_{\bar{x}} / \epsilon$ and integrating once yields, after dropping the bars,

$$
\begin{array}{cl} 
& \frac{\delta\left(h-h_{m}\right)}{h^{3}}=\frac{d}{d x}\left[h^{\prime \prime}(x)-\mu_{s}(h)\right] \\
\text { with } \quad & \delta=\frac{3 \mathrm{Ca}}{\epsilon^{3}}, \quad \mathrm{Ca}=\frac{\eta U}{\gamma}, \quad \mu_{s}(h)=\frac{\widehat{\mu}_{s}(h)}{\gamma \epsilon^{2}}
\end{array}
$$

where $\delta$ is the appropriately rescaled capillary number $\mathrm{Ca}$. For $\delta \ll 1$, this equation is solved separately in the microscopic and mesoscopic regions, and solutions are matched considering a 
respective subdominant term as a perturbation. Since Eq. (4) does not contain the coordinate explicitly, the order can be further reduced (for a monotonic section) by replacing the variable $y(h)=\left[h^{\prime}(x)\right]^{2}:$

$$
\pm \frac{\delta\left(h-h_{m}\right)}{\sqrt{y} h^{3}}=\frac{1}{2} y^{\prime \prime}(h)-\mu_{s}^{\prime}(h), \quad y\left(h_{m}\right)=0 .
$$

In the next sections. solutions are determined in the mesoscopic and microscopic region, respectively. For comparison, the stationary moving droplets described by Eq. (4) will also be computed numerically using continuation techniques ${ }^{45,46}$ employing the software AUTO9777.

\section{EXACT MESOSCOPIC SOLUTION}

\section{A. General solution}

At large distances $(h \gg 1)$ a simplified "mesoscopic" equation can be obtained by discarding the disjoining potential term in Eq. (4) and neglecting also $h_{m} \ll h$ in the viscous term:

$$
\delta h^{-2}=\partial_{x x x} h
$$

Rescaling the height $h=\delta^{1 / 3} \zeta$ reduces Eq. (6) to a parameterless form

$$
\zeta^{-2}=\partial_{x x x} \zeta
$$

This equation is invariant to simultaneous rescaling of $\zeta$ and $x$. We chose $\delta>0$, however, results for $\delta<0$ can be obtained by the transformation $x \rightarrow-x$.

Equation (7) has an exact solution expressed in a parametric form through Airy functions ${ }^{32}$ :

$$
\begin{aligned}
\zeta(s)=\frac{K}{\pi^{2} u^{2}(s)}, & x(s) & =\frac{2^{1 / 3} K}{u(s)}\left[\operatorname{Ai}(s) \operatorname{Bi}\left(s_{0}\right)-\operatorname{Ai}\left(s_{0}\right) \operatorname{Bi}(s)\right] \\
\text { with } & u(s) & =\operatorname{Ai}(s) \operatorname{Bi}^{\prime}\left(s_{0}\right)-\operatorname{Ai}^{\prime}\left(s_{0}\right) \operatorname{Bi}(s) .
\end{aligned}
$$

An indefinite factor $K$ appears here due to scale invariance of Eq. (7). It corresponds to the height of the droplet expressed in units of $h_{m}$, i.e. it has to be large.

The parametric solution (8), generally, defines a discontinuous function $\zeta(x)$, which is physically relevant only within certain intervals. For $s_{0}<s^{\dagger}$, where $s^{\dagger} \approx-1.01879$ is the largest zero of $\operatorname{Ai}^{\prime}(s)$, physically irrelevant solutions arise with $\zeta \rightarrow \infty$ for $x \rightarrow \pm \infty$ and a minimum in between. For $s^{\dagger}<s_{0}$ solutions exist with $h \rightarrow 0$ at $s \rightarrow \infty$, which correspond to a sharp receding contact line at

$$
x^{\star}=2^{1 / 3} K \operatorname{Ai}\left(s_{0}\right) / \operatorname{Ai}^{\prime}\left(s_{0}\right)<0 .
$$




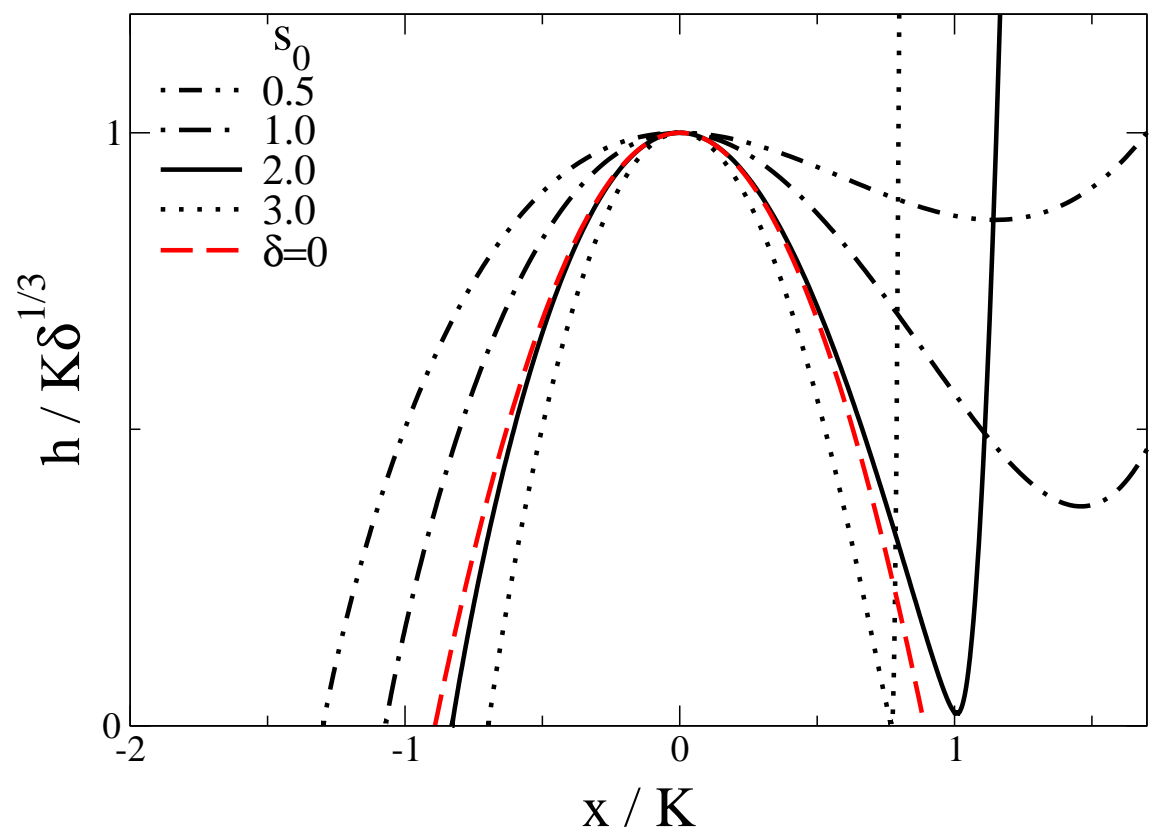

FIG. 1: Droplet profiles on the mesoscale given by Eq. (8) for different $0 \geq s_{0}$ as given in the legend. The $y$ axis represents the height as $\zeta(x) / K$, i.e. corresponding to $h / K \delta^{1 / 3}$ in the scaling used on the microscale. For comparison, we also give the parabolic shape of a static droplet on a homogeneous substrate. The droplet has the same volume $\int \zeta(x) d x$ as the one for $s_{0}=2$.

For $s^{\dagger}<s_{0}<0$ the height $\zeta$ increases monotonically with $x$. These solutions are used as a model for a receding contact line in Ref.28. If, however, $s_{0}>0$, the profile $\zeta(x)$ has a maximum at $s=s_{0}$ corresponding to $x=0$; the solutions $\zeta(x)$ pass through a minimum at $s_{\min }<s_{0}$, i.e. $x_{\text {min }}>0$, before diverging as $\zeta \sim x^{2}, x \rightarrow \infty$ at $s=s^{\star}$, where $s^{\star}\left(s_{0}\right)<0$ is the largest zero of $u(s)$. As $s_{0}$ increases, the minimum comes very close to the $x$ axis and the curvature at the minimum becomes very large. Examples of solutions for different $0 \leq s_{0}$ are shown in Fig 1

\section{B. Physically relevant interval}

We focus here on the case of moderately large $s_{0}$ taking the profile between $\zeta=0$ for $x=x^{\star}$ and the minimum of $\zeta(x)$ at $x=x_{\min }$ as the outer solution for a moving droplet driven by a force fed in on the microscale. The two parameters $s_{0}$ and $K$, as well as the droplet velocity that is absorbed into the scaling, should be obtained by matching the two inner (microscopic) solutions at advancing and receding sides, as well as fixing the droplet volume.

For moderately large $s_{0}$ one finds that the location of the minimum closely approaches $s_{\min }=$ 


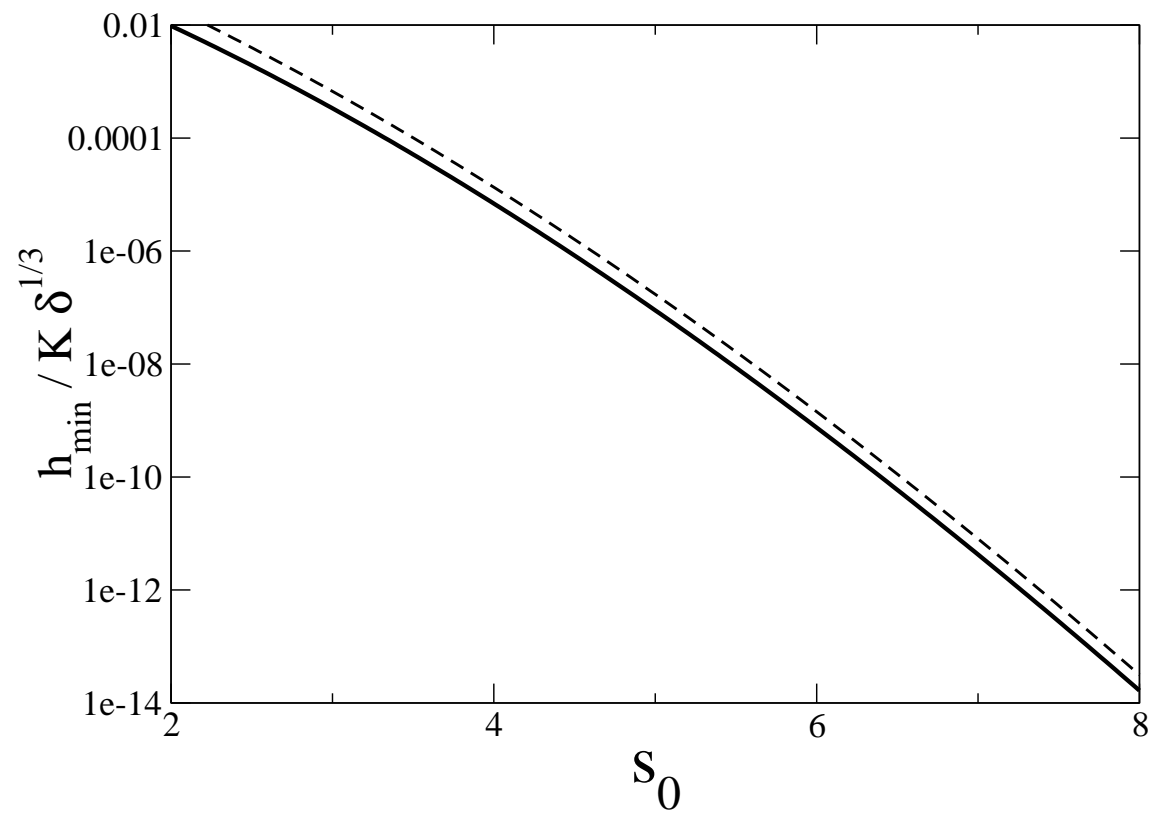

FIG. 2: The residual height at the minimum of the droplet profiles as a function of $s_{0}$. The ordinate represents the minimal height as $\zeta_{\min } / K$, i.e. corresponding to $h_{\min } / K \delta^{1 / 3}$ in the scaling used on the microscale. The solid line gives the result using the approximative $s_{\min }=-1.01879$ valid for moderately large $s_{0}$ [Eq. [15]. The dashed line gives the result using the full Eq. (8).

-1.01879 , which is the largest zero of $\mathrm{Ai}^{\prime}(s)$. This follows from asymptotic relations applicable at moderately large $s_{0}$ that will be further discussed in Section $\amalg$ Th The resulting residual profile height at the minimum is plotted in Fig. 2. For a physical precursor film thickness $h_{m}$ of the order of $1 \mathrm{~nm}$ for a millimetric drop $K=10^{6}$ and one needs $s_{0} \approx 4.5$. For a droplet of one micron height $K=10^{3}$ and $s_{0} \approx 2.5$.

Using the asymptotics of Airy functions at $s \rightarrow \infty$,

$$
\operatorname{Ai}(s) \asymp \mathrm{e}^{-\frac{2}{3} s^{3 / 2}} s^{-1 / 4}\left[\frac{1}{2 \sqrt{\pi}}+O\left(s^{-2}\right)\right], \quad \operatorname{Bi}(s) \asymp \mathrm{e}^{\frac{2}{3} s^{3 / 2}} s^{-1 / 4}\left[\frac{1}{\sqrt{\pi}}+O\left(s^{-2}\right)\right],
$$

the solution can be expanded near the zero of $\zeta$ as

$$
\begin{aligned}
& x(s) \asymp \frac{2^{1 / 3} K}{\operatorname{Ai}^{\prime}\left(s_{0}\right)}\left\{\operatorname{Ai}\left(s_{0}\right)+\frac{\mathrm{e}^{-\frac{4}{3} s^{3 / 2}}}{2 \pi \mathrm{Ai}^{\prime}\left(s_{0}\right)}\left[1+O\left(s^{-3 / 2}\right)\right]\right\}+O\left(\mathrm{e}^{-\frac{8}{3} s^{3 / 2}}\right), \\
& \zeta(s) \asymp \frac{K \sqrt{s} \mathrm{e}^{-\frac{4}{3} s^{3 / 2}}}{\pi\left[\mathrm{Ai}^{\prime}\left(s_{0}\right)\right]^{2}}\left[1+O\left(s^{-3 / 2}\right)\right]+O\left(\mathrm{e}^{-\frac{8}{3} s^{3 / 2}}\right),
\end{aligned}
$$

Explicit asymptotics is obtained by solving Eq. (11) with respect to $s$ :

$$
s \asymp\left(-\frac{3}{4} \ln \frac{x-x^{\star}}{L}\right)^{2 / 3}\left[1+O\left(\ln ^{-2} \frac{x-x^{\star}}{L}\right)\right] .
$$


where $L^{-1}=2^{2 / 3} \pi \mathrm{Ai}^{\prime}\left(s_{0}\right)^{2} / K$. This yields, up to corrections of higher order in $\ln \left[\left(x-x^{\star}\right) / L\right]$,

$$
\zeta(x) \asymp\left(x-x^{\star}\right)\left(-3 \ln \frac{x-x^{\star}}{L}\right)^{1 / 3}, \quad \zeta^{\prime}(x) \asymp\left(-3 \ln \frac{x-x^{\star}}{L}\right)^{1 / 3} .
$$

The length $L$ is very large for moderately large $s_{0}$.

\section{Limit of weak driving}

Although Eq. (7) does not contain the rescaled capillary number $\delta$, we expect the applicable outer solution to become symmetric, approaching a parabolic profile, in the limit $\delta \rightarrow 0$, which corresponds to a vanishing wettability gradient. As illustrated in Fig.1 and confirmed by the following asymptotic analysis, the outer solution becomes almost symmetric at large values of $s_{0}$, which, as we shall further see, correspond to small values of $\delta$.

The limit $s_{0} \rightarrow \infty$ can be obtained with the help of the asymptotics (10) of Airy functions, which is practically applicable already at moderately large values $s_{0}>2$. The resulting asymptotic profile height at the minimum on the advancing edge is

$$
\zeta_{\text {min }} \asymp K\left[\pi \mathrm{Ai}\left(s_{\text {min }}\right) \mathrm{Bi}^{\prime}\left(s_{0}\right)\right]^{-2} \asymp 1.10937 K s_{0}^{-1 / 2} \mathrm{e}^{-\frac{4}{3} s_{0}^{3 / 2}}\left[1+O\left(s_{0}^{-3 / 2}\right)\right],
$$

The minimum is located, up to an exponentially small correction proportional to $\mathrm{e}^{-\frac{4}{3} s_{0}^{3 / 2}}$, at the largest zero of $\mathrm{Ai}^{\prime}(s)$, i.e. $s_{\min }=-1.01879$ (see Fig. 2).

The asymptotic expression for the second derivative $c=\zeta^{\prime \prime}(x)$ at the minimum is

$$
\begin{aligned}
c\left(s_{\text {min }}\right) & \asymp 2^{1 / 3} K^{-1} \pi^{2}\left[\operatorname{Ai}^{\prime}\left(s_{\text {min }}\right)^{2}-s_{\text {min }} \operatorname{Ai}\left(s_{\text {min }}\right)^{2}\right] \operatorname{Bi}^{\prime}\left(s_{0}\right)^{2} \\
& \asymp \widehat{c} K^{-1} s_{0}^{1 / 2} \mathrm{e}^{\frac{4}{3} s_{0}^{3 / 2}}\left[1+O\left(s_{0}^{-3 / 2}\right)\right] .
\end{aligned}
$$

with $\widehat{c} \approx 1.15697$.

The corresponding asymptotic value of the coordinate $x$ is

$$
x_{\text {min }} \asymp 2^{1 / 3} K s_{0}^{-1 / 2}\left[1+\frac{1}{4} s_{0}^{-3 / 2}+O\left(s_{0}^{-3}\right)\right],
$$

In the leading order, this coincides by the absolute value with the asymptotics of $x^{\star}$ given by Eq. (9):

$$
x^{\star} \asymp-2^{1 / 3} K s_{0}^{-1 / 2}\left[1-\frac{1}{4} s_{0}^{-3 / 2}+O\left(s_{0}^{-3}\right)\right] .
$$

This points out to the symmetry that should be attained in the limit of zero velocity. The full profile away from the location of the minimum should be computed by assuming both $s_{0}$ and $s$ to 
be large. This yields, in the leading order,

$$
\zeta \asymp K\left(s / s_{0}\right)^{1 / 2} \operatorname{sech}^{2}\left[\frac{2}{3}\left(s_{0}^{-3 / 2}-s^{-3 / 2}\right)\right], \quad x \asymp 2^{1 / 3} K s_{0}^{-1 / 2} \tanh \left[\frac{2}{3}\left(s_{0}^{-3 / 2}-s^{-3 / 2}\right)\right] .
$$

At the receding edge, the asymptotics of these expressions at $s \gg s_{0}$ coincides with the asymptotics of Eqs. (11), (12) at large $s_{0}$.

The height is of the same order of magnitude as the macroscopic length scale $K$ only when $s$ is close to $s_{0}$. Setting $s=s_{0}$, one can see by combining the above expressions that the function $\zeta(x)$ indeed approaches in this limit the parabolic profile $\zeta / K=1-b^{2} x^{2}$ with $b=2^{-1 / 3} s_{0}^{1 / 2} / K$ everywhere except the immediate vicinity of both contact lines; the corrections are of $O\left(s_{0}^{-2}\right)$. Thus, the scaled droplet volume is computed as

$$
V=2 K \delta^{1 / 3} \int_{0}^{1 / b}\left(1-b^{2} x^{2}\right) \mathrm{d} x=\frac{4 K \delta^{1 / 3}}{3 b}=\frac{4(2 \delta)^{1 / 3} K^{2}}{3 s_{0}^{1 / 2}} .
$$

\section{MICROSCOPIC SOLUTION AND MATCHING}

\section{A. Expansion in $\delta$}

In the microscopic region, the thickness changes from $h=h_{m}$ to a "mesoscopic" value far exceeding $h_{m}$ but small compared to the drop size and capillary length (that is here infinite). Solving Eq. (4) with $\delta=0$ defines the static contact angle in the limit $h \rightarrow \infty$, while for $\delta \neq 0$ an apparent dynamic contact angle is obtained in this limit. The appropriate length scale in this region is $h_{m}$; the respective dimensionless form of Eqs. (4) and (5) is

$$
\begin{aligned}
& \delta \frac{h-1}{h^{3}}=\frac{d}{d x}\left[h^{\prime \prime}(x)-\mu_{s}(h)\right], \\
& \pm \delta \frac{h-1}{\sqrt{y} h^{3}}=\frac{1}{2} y^{\prime \prime}(h)-\mu_{s}^{\prime}(h),
\end{aligned}
$$

with

$$
\mu_{s}(h)=\frac{\beta^{2}}{h^{3}}\left(1-\frac{1}{h^{3}}\right), \quad \beta=\frac{1}{\epsilon h_{m}} \sqrt{\frac{Q_{s}}{\gamma}}
$$

To model different wettability at the advancing and receding contact line one assumes different constants $\beta=\beta_{a d v}$ and $\beta=\beta_{\text {rec }}$, respectively. A higher wettability at the advancing side is assured by $\beta_{a d v}<\beta_{\text {rec }}$.

For the receding meniscus, the positive sign should be chosen in Eq. (22), and the boundary conditions are $h=1, h^{\prime}(x)=0$ at $x \rightarrow-\infty$, and $h^{\prime \prime}(x) \rightarrow 0$ at $x \rightarrow \infty$, or $y(h)=0$ at $h=1$ and 
$y^{\prime}(h) \rightarrow 0$ at $h \rightarrow \infty$. The latter condition, suggested by Eggers ${ }^{28}$, should fit the curvature of the mesoscopic solution, which, according to Eq. (14), approaches $-\infty$ in the limit $x \rightarrow x^{\star}$.

For the advancing meniscus, the negative sign should be chosen. The boundary condition $h^{\prime}(x)=0$ should be set at $x \rightarrow \infty$, and the condition $h^{\prime \prime}(x) \rightarrow 0$ at $x \rightarrow-\infty$.

The solution of Eq. (22) is sought for as an expansion in $\delta: y(h)=y_{0}(h)+\delta y_{1}(h)+\ldots$. The zero-order equation,

$$
\frac{1}{2} y_{0}^{\prime \prime}(h)-\mu_{s}^{\prime}(h)=0
$$

is readily integrated to obtain

$$
y_{0}(h)=\frac{3}{5} \beta^{2} \frac{(h-1)^{2}}{h^{5}}\left(\frac{2}{3}+\frac{4}{3} h+2 h^{2}+h^{3}\right),
$$

The equilibrium contact angle $\theta_{0}$ is obtained from the zero-order equation (25) in the limit $h \rightarrow \infty$ :

$$
\theta_{0}=h^{\prime}(\infty)=\sqrt{y_{0}(\infty)}=\sqrt{3 / 5} \beta
$$

The formal small parameter $\epsilon$ can now be identified with the small physical equilibrium contact angle, say, $\widehat{\theta}_{0}^{r e c}$ and expressed through physical parameters by requiring $\widehat{\theta}_{0}^{r e c} / \epsilon=\theta_{0}^{r e c}=1$. This yields

$$
\beta_{r e c}=\sqrt{\frac{5}{3}}, \quad \epsilon=\widehat{\theta}_{0}^{r e c}=\frac{1}{h_{m}}\left(\frac{3}{5} \frac{Q_{s}^{r e c}}{\gamma}\right)^{1 / 2} \propto \frac{d}{h_{m}}\left(\frac{Q_{s}^{r e c}}{Q_{l}}\right)^{1 / 2} .
$$

The latter estimate follows from the estimate for surface tension $\gamma \propto Q_{l} / d^{2}$, where $Q_{l}$ is a characteristic interaction energy of fluid molecules and $d<h_{m}$ is the nominal molecular diameter. The contact angle is indeed small when $Q_{s}^{r e c} / Q_{l}$ (the dimensionless Hamaker constant at the advancing contact line) is small. The numerical value of $\beta_{\text {rec }}$ is specific to the particular expression for the disjoining potential (2), but the general procedure would be the same for any potential of a similar shape. Note, that now only $\beta_{a d v}<\sqrt{5 / 3}$ determines the driving wettability gradient.

Further derivation is carried out separately for receding and advancing menisci, in view of different boundary conditions for the two cases.

\section{B. Receding meniscus}

The first-order equation derived from Eq. (22) is

$$
\frac{h-1}{\sqrt{y_{0}(h)} h^{3}}=\frac{1}{2} y_{1}^{\prime \prime}(h)
$$


Using here Eq. (25) and integrating from $h=1$ to $\infty$ yields the value of $y_{1}^{\prime}(1)$ necessary to satisfy the asymptotic boundary condition of vanishing curvature at $h \rightarrow \infty$ for the receding meniscus:

$$
\begin{aligned}
q_{1} \equiv y_{1}^{\prime}(1) & =-2 \sqrt{\frac{5}{3}} \frac{1}{\beta} \int_{1}^{\infty}\left[h\left(\frac{2}{3}+\frac{4}{3} h+2 h^{2}+h^{3}\right)\right]^{-1 / 2} \mathrm{~d} h \\
& \approx-1.3383 \sqrt{\frac{5}{3}} \frac{1}{\beta}=-1.3383 .
\end{aligned}
$$

The latter value corresponds to the scaling (27).

A non-zero value of $y_{1}^{\prime}(1)$ appears to change qualitatively the character of decay to the equilibrium precursor thickness at very small deviations $h-1 \leq O(\delta)$. At these distances, the expansion, in fact, breaks down, but the solution can be readily found by linearizing Eq. (21) near $h=1$. The linear equation is solved by a combination of exponents $\mathrm{e}^{\lambda x}$ where $\lambda$ is a positive root of $\lambda^{3}-3 \beta^{2} \lambda-\delta=0$. While for $\delta=0$ the layer thickness decays at $x \rightarrow-\infty$ to unity as $\mathrm{e}^{\sqrt{3} \beta x}$, for $\delta \neq 0$ an additional small $\operatorname{root} \lambda=\delta \beta^{-2} / 3+O\left(\delta^{2}\right)$ appears. This root is positive, indicating a very slow decay to the equilibrium precursor thickness (and, possibly, breakdown of quasistationary approximation) at $\delta \rightarrow 0$.

Since $y_{1}^{\prime}(h) \sim h^{-1}$ at $h \rightarrow \infty, y_{1}(h)$ diverges logarithmically in the outer limit. The asymptotic expression is obtained by integrating Eq. (28) with the boundary condition (29):

$$
y_{1} \asymp-2 \ln \frac{h}{a_{1}}, \quad a_{1} \approx 0.444 .
$$

The respective expansion for the slope $h^{\prime}(x)$ useful for further matching to an outer solution is

$$
h^{\prime}(x) \asymp 1-\delta \ln \frac{x}{a_{1}}+O\left(\delta^{2}\right) .
$$

The expansion can be routinely continued to higher orders with the help of a symbolic computation program.

To match the mesoscopic and microscopic solutions at the receding side, we compare the outer limit of the receding microscopic solution $(h \rightarrow \infty)$ with the inner limit of the mesoscopic solution $(\zeta \rightarrow 0)$. This translates to comparing $\left[\zeta^{\prime}(x)\right]^{3}$ given by Eq. (14) with that given by Eq. (31). After rescaling Eq. (14) and shifting the location of the contact line to zero and rearranging Eq. (31), this gives

$$
\left[h^{\prime}(x)\right]^{3}=-3 \delta \ln \frac{x}{L}=-3 \delta \ln \frac{\mathrm{e}^{-(1 /(3 \delta)} x}{a_{1}} .
$$

The matching requirement yields the dependence of $L$ and, hence of $s_{0}$ on $\delta$, expressed in an implicit form

$$
L^{-1}=\frac{2^{2 / 3} \pi}{K} \operatorname{Ai}^{\prime}\left(s_{0}\right)^{2} \approx \frac{1}{2^{4 / 3} K \sqrt{s_{0}}} \exp \left[-\frac{4}{3} s_{0}^{3 / 2}\right]=a_{1} \exp \left[-\frac{1}{3 \delta}\right] .
$$


The approximate expression is valid for $s_{0} \gg 1$ (practically, for $s_{0} \gtrsim 2$ ). This expression connects $s_{0}$ with the dimensionless velocity $\delta$ as shown for different $K$ in Fig. 3

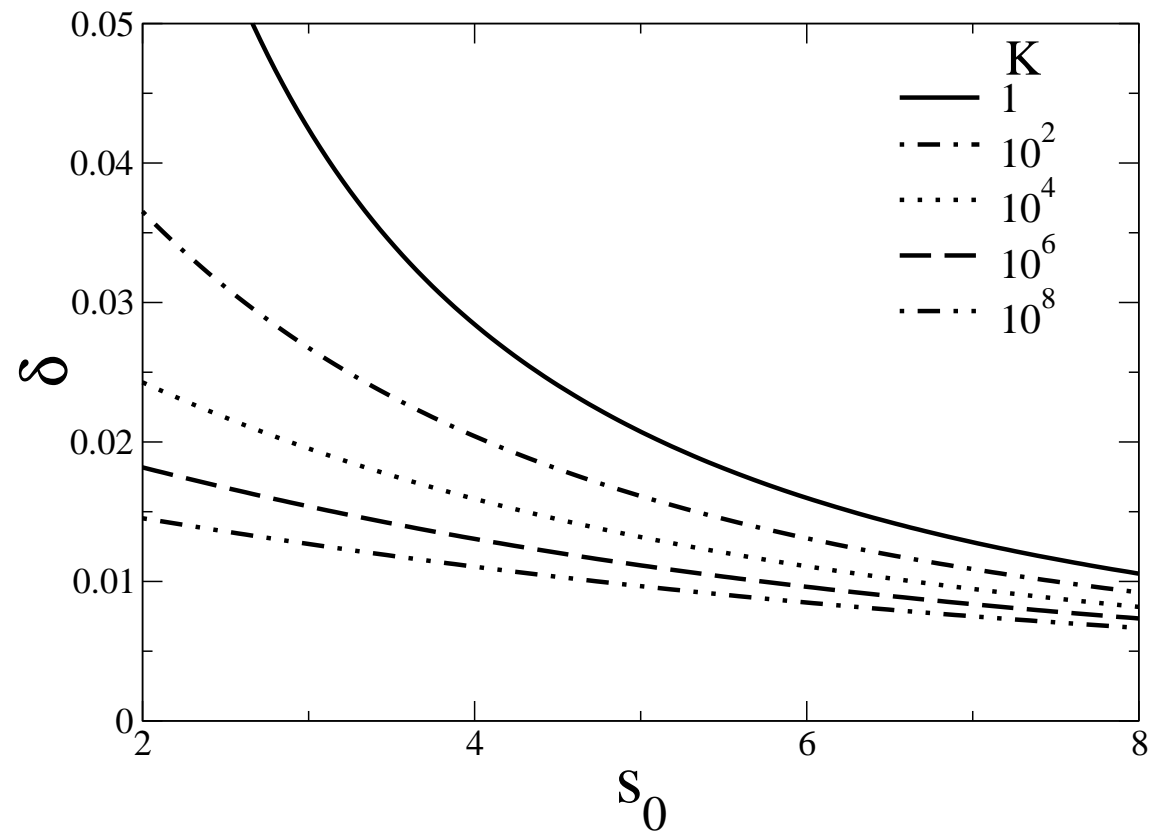

FIG. 3: The dependence of the rescaled capillary number $\delta$ on the parameter $s_{0}$ for different droplet sizes parametrized by $K$ (given in the legend) as given by Eq. (33).

Combining this result with the dependence of $s_{0}$ on droplet size discussed at Fig.2 gives an estimation of the velocity $\delta$. For a physical precursor film thickness $h_{m}$ of the order of $1 \mathrm{~nm}$ for a millimetric drop $K=10^{6}, s_{0} \approx 4.5$ and in consequence $\delta \approx 0.01$ For a droplet of one micron height $\delta \approx 0.025$.

Note that this is still only an order-of-magnitude estimate, because $K$ itself depends in a subtle way on the velocity. This can be seen in Fig. 4 where the dependency of the droplet volume

$$
V=\delta^{1 / 3} K^{2} \int_{\infty}^{s_{\min }} \zeta(s) x^{\prime}(s) d s
$$

on $s_{0}$ is plotted. To compare droplets of identical volume for different driving forces, one has to determine $K$ using the matching at the advancing edge.

\section{Advancing meniscus}

For an advancing contact line, the mesoscopic solution has no logarithmic asymptotics, and for matching one can use the zero-order microscopic solution, matching its limit at $h \rightarrow \infty$, 


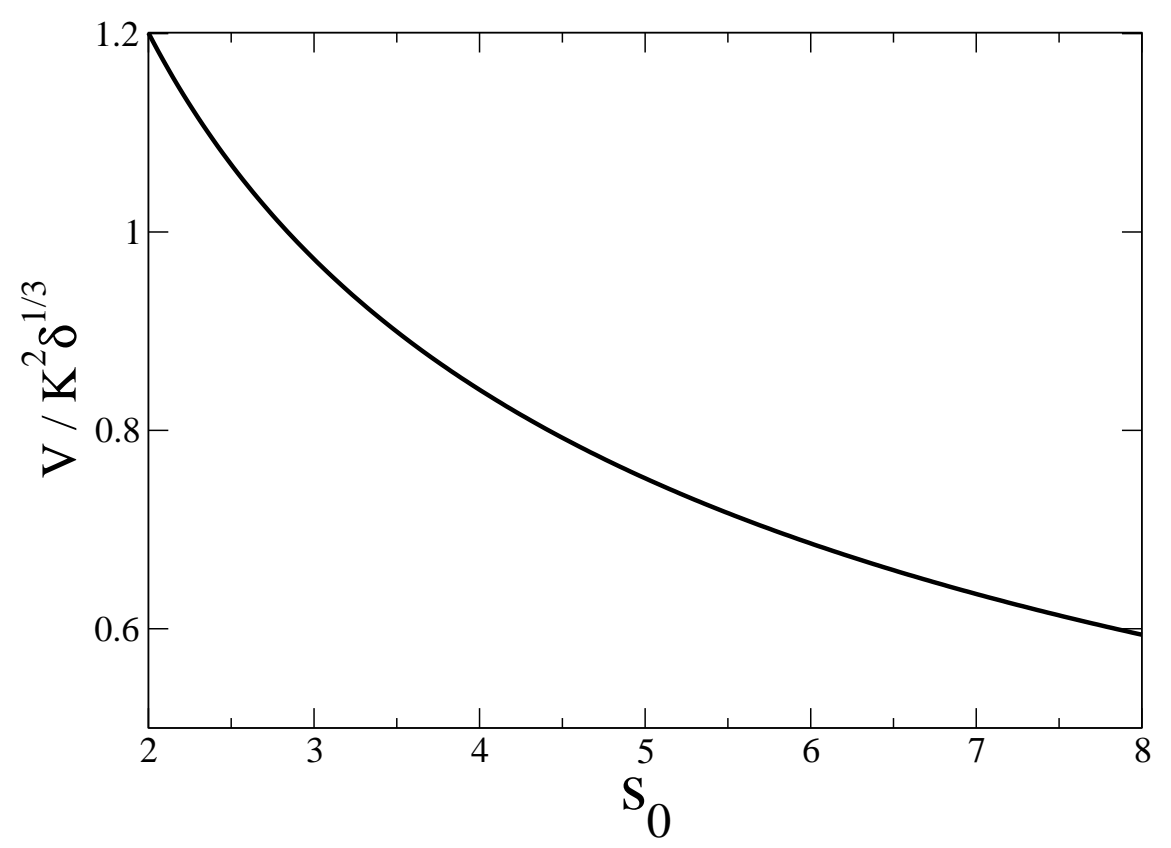

FIG. 4: Dependence of the droplet volume on the parameter $s_{0}$. The $y$-axis represents the scaled volume $V / K^{2} \delta^{1 / 3}$ with $V$ given by Eq. 34. $V / \theta_{0}$ represents a physical volume that may be used as a fixed control parameter. The numerical result using the minimum calculated with the full Eq. 8 as integration boundary can not be distinguished from the solid line.

$h^{\prime \prime}(x) \rightarrow 0$ to the mesoscopic solution at the inflection point $\zeta^{\prime \prime}(x)=0$.

This translates to comparing $\delta^{1 / 3} \partial_{x} \zeta\left(s_{i}\right)=\delta^{1 / 3} \zeta^{\prime}\left(s_{i}\right) / x^{\prime}\left(s_{i}\right)$ at the inflection point $s=s_{i}$ given by

$$
s_{i}\left[\operatorname{Ai}^{\prime}\left(s_{0}\right) \operatorname{Bi}\left(s_{i}\right)-\operatorname{Ai}\left(s_{i}\right) \operatorname{Bi}^{\prime}\left(s_{0}\right)\right]^{2}=\left[\operatorname{Ai}^{\prime}\left(s_{0}\right) \operatorname{Bi}^{\prime}\left(s_{i}\right)-\operatorname{Ai}^{\prime}\left(s_{i}\right) \operatorname{Bi}^{\prime}\left(s_{0}\right)\right]^{2}
$$

to $h^{\prime}(x \rightarrow \infty)=\theta_{0}^{\text {adv }}$ defined by Eq. (26) with $\beta$ replaced by $\beta_{a d v}$. As result of the matching, one finds

$$
\delta=\left(\frac{\theta_{0}^{a d v}}{\partial_{x} \zeta\left(s_{i}\right)}\right)^{3},
$$

i.e. $\delta /\left(\theta_{0}^{a d v}\right)^{3}$ can be calculated as a function of the parameter $s_{0}$ as presented in Fig. 5 ,

This procedure effectively cuts off the highly curved segment of the mesoscopic solution near the minimum. Take note that $\zeta_{i}$ is still much larger than the $O(1)$ microscopic scale, and one can expect corrections due to the disjoining potential to become significant only well below this value. However, as we will illustrate in the Conclusion, the first order matching is already sufficient to completely describe the droplets driven by a wettability gradient. 


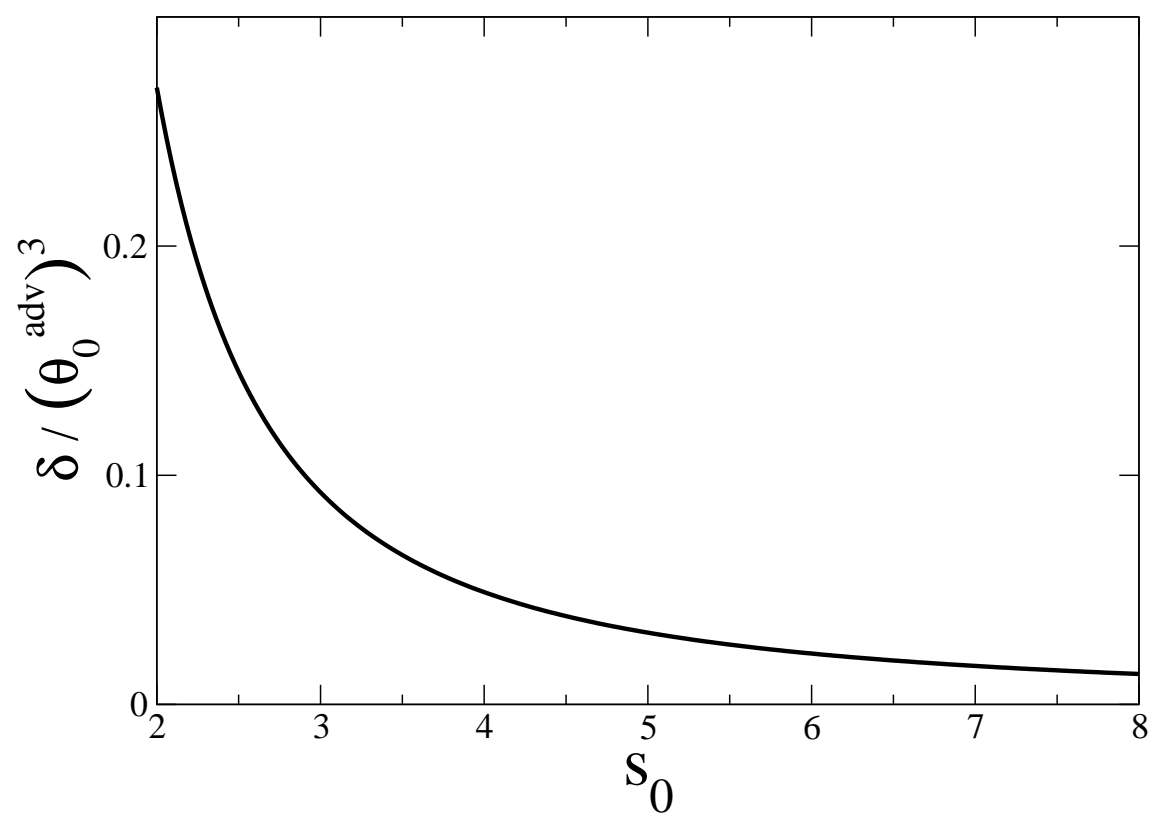

FIG. 5: Dependence of the scaled droplet velocity $\delta /\left(\theta_{0}^{a d v}\right)^{3}$ on the parameter $s_{0}$, as obtained from the inflection point matching at the advancing contact zone [Eq. [36].

\section{CONCLUSION}

We have developed an asymptotic theory for a moving drop driven by a wettability gradient. Wide separation between the meso- and microscale allows us to use respective analytical and expanded solutions on the different scales.

Matching of the mesoscale and microscale solutions at the advancing and the receding contact region allows to obtain the droplet shape and the velocity of movement as functions of the imposed wettability gradient and droplet volume. In this way, the two matching procedures together with a translation between the different scalings gives a complete characterization of the droplet motion for a given physical volume $V / \theta_{0}$ and the wettability gradient characterized by the physical receding $\hat{\theta}_{0}^{r e c}=\theta_{0} \theta_{0}^{r e c}=\theta_{0}$ and advancing $\hat{\theta}_{0}^{a d v}=\theta_{0} \theta_{0}^{a d v}<\theta_{0}$ equilibrium angle.

Fixing the overall long-wave scaling by fixing $\theta_{0}$, the three relations between $\theta_{0}^{a d v}, V, \delta, K$ and $s_{0}$ obtained in the course of the present work [Eqs. (33), (34) and (36) illustrated in Figs. 3 4 and 5] respectively] allow us to determine the unknown $\delta, K$ and $s_{0}$ for each given pair of $\theta_{0}^{a d v}$ and $V$. In Fig. 6 results of the asymptotic matching are given for the velocity $\delta$, and the "shape parameter" $s_{0}$ in dependence of the advancing equilibrium contact angle $\theta_{0}^{a d v}$ for a selection of volumes $V$. As expected, the velocity goes towards zero as the driving wettability difference 


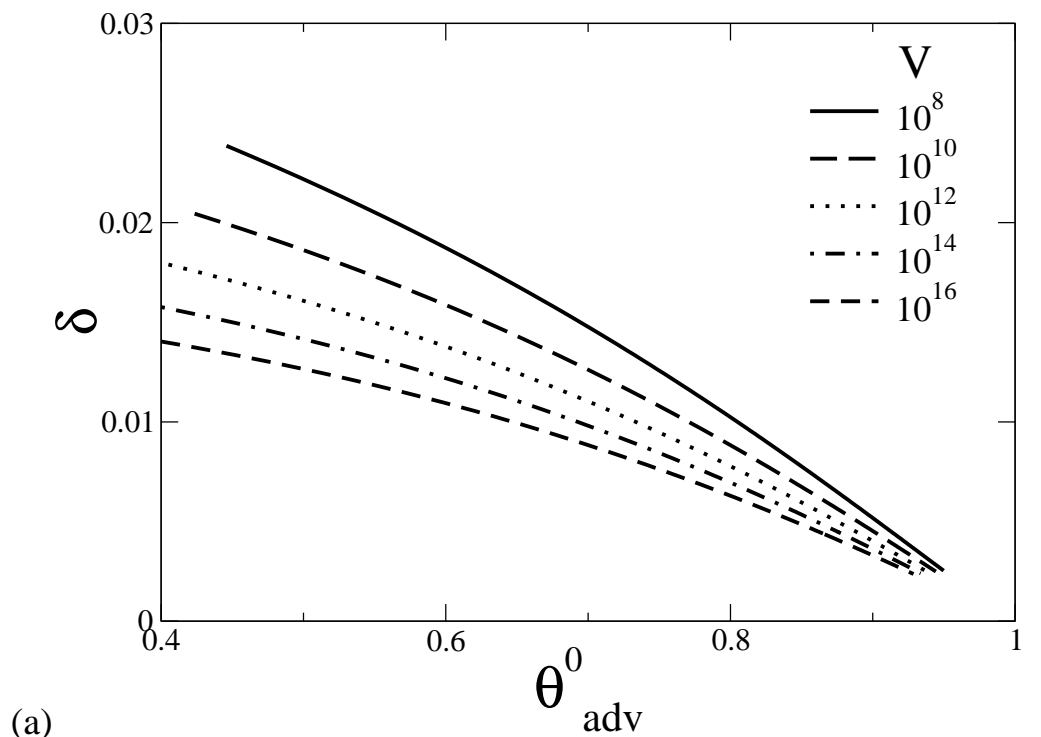

(a)

(b)

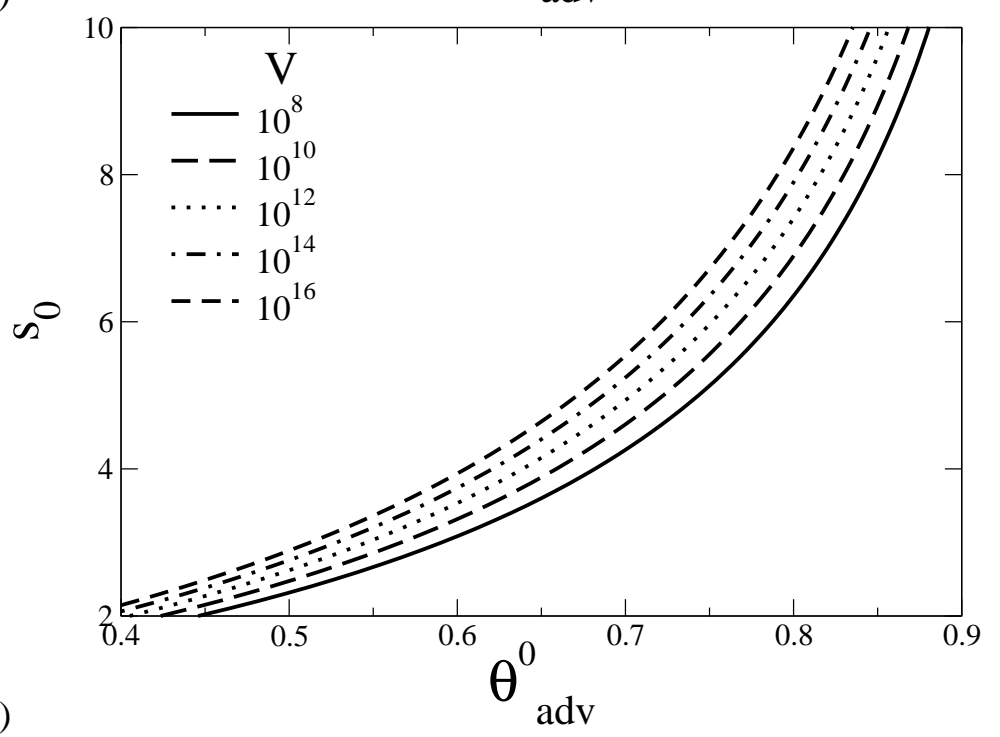

FIG. 6: Results of the numerical matching proceedure described in the main text using at the advancing contact zone the inflection point matching. Shown are (a) the droplet velocity $\delta$, and (b) the parameter $s_{0}$ describing the mesoscopic shape in their dependency on the imposed equilibrium advancing contact angle $\theta_{a d v}<\theta_{\text {rec }}=1$ for different given droplet volumes $V$ as given in the legend. In (a) results are shown for $2 \leq s_{0} \leq 20$.

$\theta_{0}^{r e c}-\theta_{0}^{a d v}$ vanishes, i.e. $\theta_{0}^{r e c} \rightarrow 1$. The shape parameter $s_{0}$ diverges for $\theta_{0}^{r e c} \rightarrow 1$ as discussed in section III At a fixed driving $\theta_{0}^{a d v}$, the droplet becomes more asymmetrical ( $s_{0}$ decreases) and faster with decreasing volume. The velocity changes with volume are more pronounced for larger driving (i.e. smaller $\theta_{0}^{a d v}$ ).

Albeit the matching is based on an expansion in $\delta$, the numerical calulations leading to Fig.6 
are not practical for very small $\delta$ (i.e. large $s_{0}$ ) because already for $s_{0}=20$ the calculation involves small numbers of the order of $e^{-120}$ that are difficult to handle. Under these conditions, the shape, however, remains almost static, and the integral relations of Ref. 48 can be used. The relations obtained by multiplying Eq. (4) by $h-h_{m}$ and integrating over the entire $x$ axis yield the expression for the dimensionless velocity in the form of a ratio $\delta=F / I$ of the driving force $F$ to the dissipative integral

$$
I=2 \ln \frac{2 a}{b h_{m}},
$$

where $a=(3 / 2 V)^{1 / 2}$ is the radius of a static parabolic droplet with the profile $h=\frac{1}{2} a\left[1-(x / a)^{2}\right]$ and $b \approx 2.082$ is a constant. The driving force $F=F^{r e c}-F^{a d v}$ is expressed through the equilibrium contact angles by separating the contributions of the two menisci $F^{r e c}, F^{a d v}$ :

$$
F=-\int_{-\infty}^{\infty}\left(h-h_{m}\right) \frac{d \mu_{s}}{d x} d x=\int_{-\infty}^{\infty} \mu_{s} \frac{d h}{d x} d x=F^{r e c}-F^{a d v}
$$

where, after replacing the integration variable and extending integration to infinity in a thick middle part of the droplet where the disjoining potential is negligible,

$$
F^{r e c, a d v}=\int_{h_{m}}^{\infty} \mu_{s}^{r e c, a d v}(h) d h=\frac{\widehat{\theta}_{r e c, a d v}^{2}}{2} .
$$

This yields (with $\theta_{\text {rec }}=1$ )

$$
\delta=\frac{1-\theta_{a d v}^{2}}{4 \ln \left(2 a / b h_{m}\right)} .
$$

This result is compared to the asymptotic theory in Fig 7

The presented asymptotic theory is based on (i) a separation into micro- and mesoscale, i.e. it is not valid for $V$ too small ( $s_{0}$ becomes too small) and (ii) an expansion in $\delta$, i.e. it is not valid for $\delta$ too large. Assuming a precursor film of $1 \mathrm{~nm}, V=10^{6}$ corresponds roughly to droplets of $1 \mu \mathrm{m}$ height, implying that the asymptotics is valid in the realm of microfluidics, but less so for nanofluidics. However, because for nanodroplets the micro- and mesoscale are not well separated they can be treated with numerical methods. It is convenient to calculate stationary moving droplets using continuation techniques ${ }^{45.46}$ as shown, for instance, for nanodroplets moving under the influence of a body force ${ }^{49.50}$ and chemically driven droplets ${ }^{41.42}$. However, the numerical calculation becomes very tedious for larger drops because of the separation of scales.

In Fig. 7 we present a comparison of asymptotic results (stretched down to $V=10^{6}$ ) obtained from Eqs. (33), (34) and (36), small- $\delta$ results given by Eq. (40), and numerical continuation results (stretched up to $V=10^{8}$ ) for Eq. (21). For small driving $\theta_{a d v}^{0}>0.8$ the overall agreement of the 


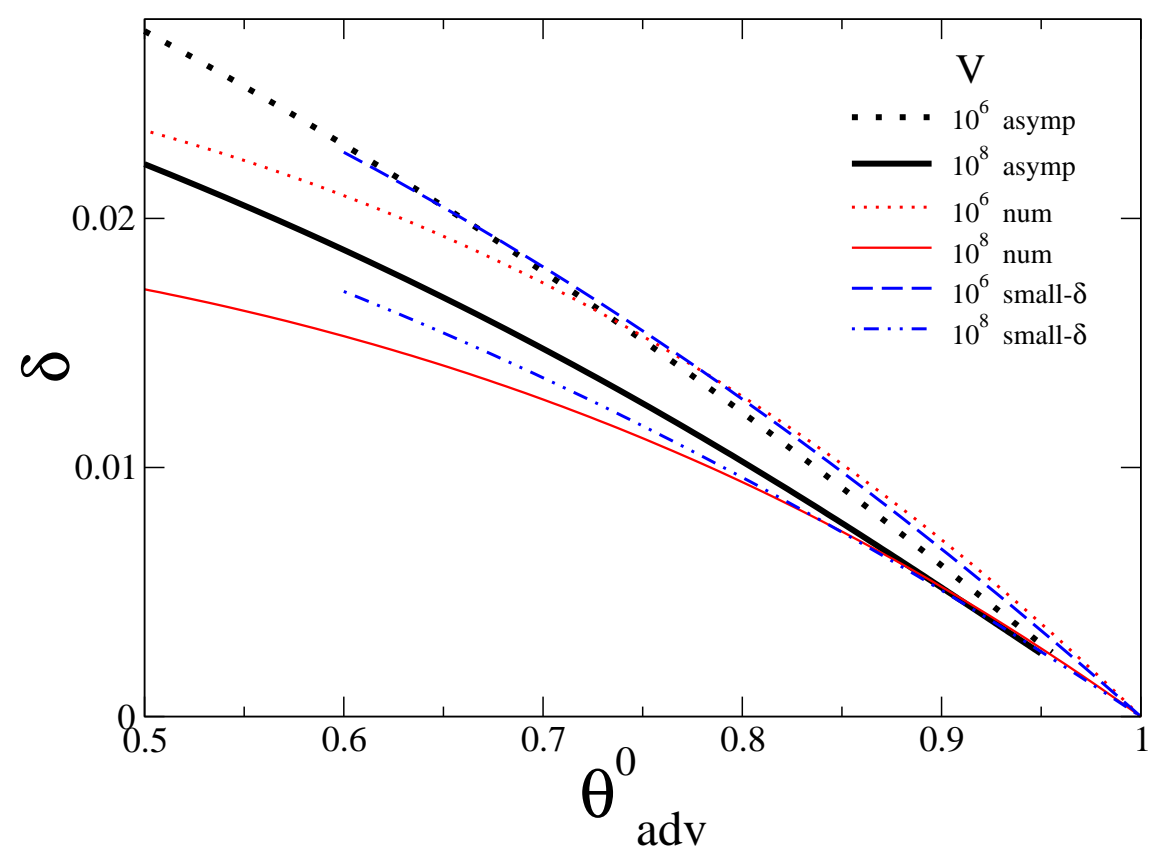

FIG. 7: Comparison of asymptotic results (thick lines) obtained from Eqs. (33), 34) and (36), numerical continuation results (corresponding thin lines) for Eq. (21), and the small- $\delta$ results given by Eq. (40) (dashed lines). The dependence of the droplet velocity $\delta$, on the imposed equilibrium contact angle at the advancing side $\theta_{a d v}<\theta_{\text {rec }}=1$ is shown for different given droplet volumes $V$, as specified in the legend.

three methods is reasonably good. For $V=10^{6}$ the maximal deviation is below $15 \%$, and for $V=10^{8}$ it is about $5 \%$. As expected, for larger driving $\theta_{a d v}^{0}<0.8$ the results start to deviate, the numerical solutions of the full Eq. (21) give a lower velocity than the asymptotics, and more so for smaller $\theta_{a d v}^{0}$. For larger droplets this deviation starts at larger $\theta_{a d v}^{0}$ (smaller driving). There are various small factors that may contribute to the deviations at small driving: (i) for $V=10^{8}$ the equilibrium contact angle still differs from the asymptotic value of one by about $0.3 \%$; (ii) for moving droplets the precursor film thickness depends weakly on the dynamics ${ }^{49.51}$ implying a droplet volume that is not exactly constant with changing velocity. For $V=10^{8}$ and $\theta_{a d v}^{0}=0.5$ the precursor film thickness is about 1.0025, i.e. for the used domain size of $10^{6}$ the relative change in droplet volume is negligible $\left(\Delta V / V \approx 10^{-5}\right)$.

Surprisingly, the simple results obtained for small $\delta$ in Ref. 48 as the ratio of the driving force and the dissipative integral [our Eq. (40)] seem to fit the numerical data better than the asymptotic theory. This results, apparently, from the cancelation of different approximations. The assumed velocity-independent parabolic droplet shape underestimates, for instance, the dissipation at the 
receding contact line and in the bulk, but overestimates the dissipation at the advancing contact line. The advantage of the asymptotic theory can be better appreciated comparing the profiles of the moving droplets (Fig, 8 ).

The numerical results obtained by continuation (solid lines) strongly differ from the static droplet shapes (dotted lines) that are the basis for the small- $\delta$ approximation Eq. (40). The asymptotic mesoscopic profiles [Eqs (11) and (12) with the parameters obtained from Eqs. (33), (34) and (36)] approach the numerical results reasonably good for weak driving $\theta_{a d v}^{0}=0.8$, independently of whether one compares profiles for identical velocity or driving (Fig 8 (a)). For larger driving, the comparison of profiles for identical velocities gives better results. In general, the receding part is described quite perfectly. The advancing part differs because the matching is based on the advancing equilibrium contact angle that is smaller than the dynamical one.

Our treatment has made it clear that the characteristics of the moving droplets depend in a crucial way on the kind of driving used. The droplet may be driven by body forces, as for instance, gravitation or Marangoni forces. In lubrication theory the latter also takes the form of a body force althought physically it acts at the free surface only. The driving is top-down because the force is fed into the system on the macroscopic scale and causes motion on all scales down to the microscale. One of our main results is that this type of driving cannot be described by the present theory because the balance of the viscous term and the capillary term in Eq. (6) does not account for the driving force. Specifically, it is not possible to use the solution of Eq. (6) in terms of Airy functions to describe droplets sliding down an incline driven by gravity. This is already obvious from the fact that for gravity-driven drops the advancing dynamic contact angle is larger than the receding one ${ }^{49,50}$ contrary to the characteristics of the mesoscopic solution given by Eqs. (11) and (12).

On the contrary, driving the droplets by a wettability gradient is bottom-up because the force is fed into the system on the microscale and causes motion up to the mesoscale (in our terms, no macroscale exists in this case because the macroscale is defined by the scale of the body forces that are absent by definition of the problem).

\section{Acknowledgments}

LMP and UT acknowledge support by the Israel Science Foundation (grant \# 55/02) and the European Union (MRTN-CT-2004-005728), respectively. 


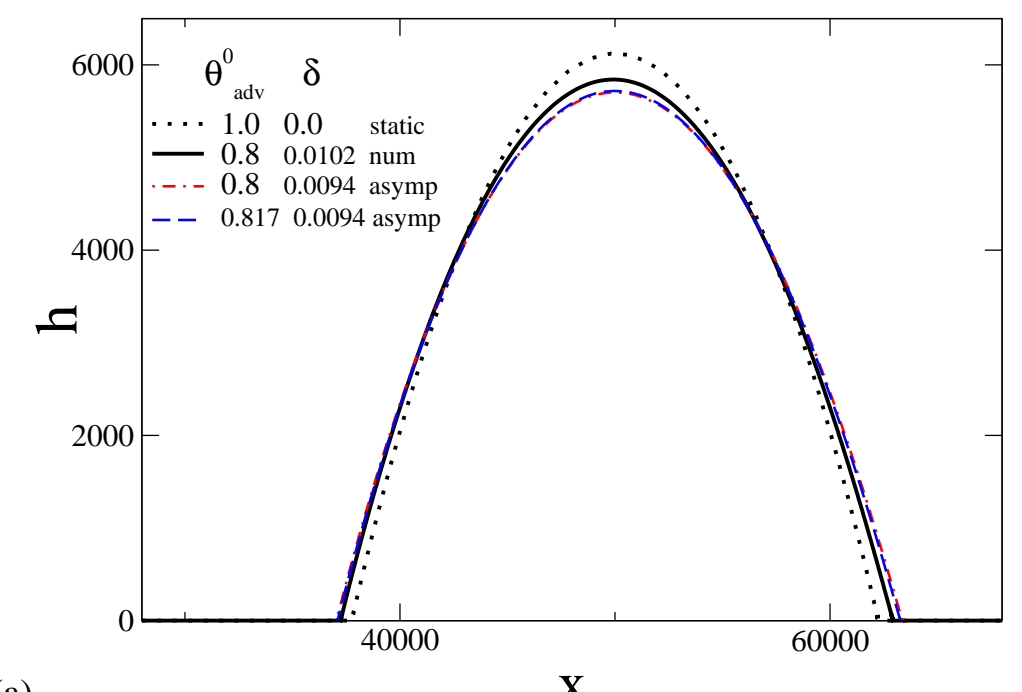

(a)

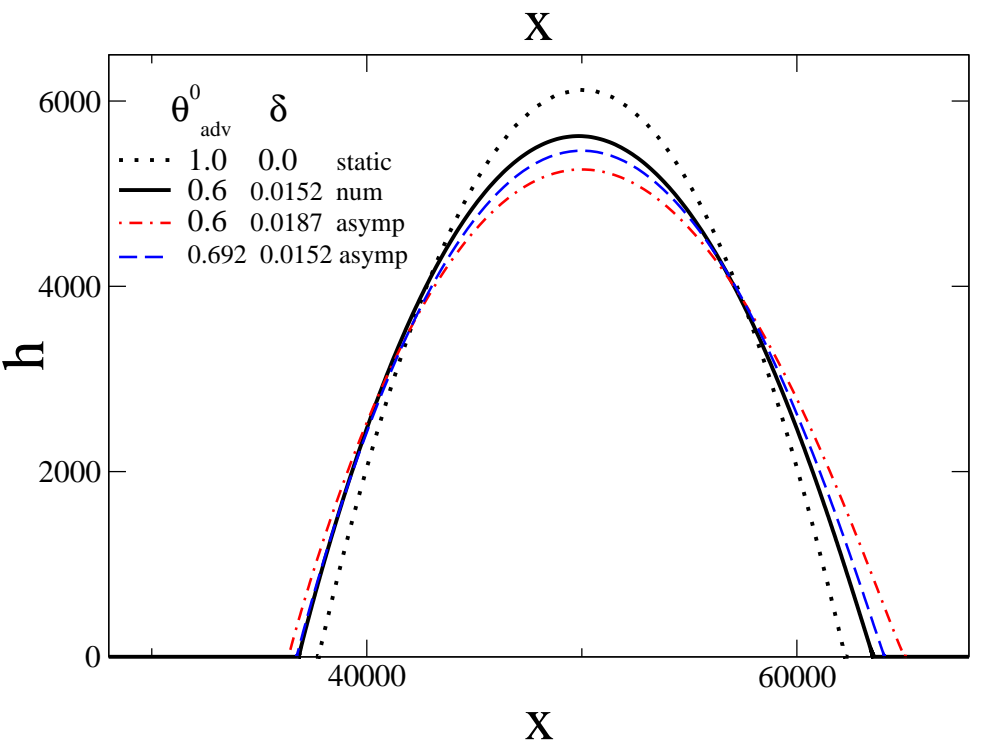

FIG. 8: Profiles of moving droplets for $V=10^{8}$ as obtained by numerical continuation of stationary solutions [Eq. (21), solid lines] are compared to the solutions of the mesoscopic asymptotic Eq. (6). For the latter profiles are given that have the same $\theta_{0}^{a d v}$ (dot-dashed lines) or the same $\delta$ (dashed line) as the numerical solution. Parameters are (a) numerical: $\theta_{0}^{a d v}=0.8, \delta=0.009$; asymptotic (dot-dashed) $\theta_{0}^{a d v}=$ $0.8, \delta=0.010, s_{0}=6.35, K / V^{1 / 2}=2.63$; asymptotic (dashed) $\theta_{0}^{a d v}=0.82, \delta=0.009, s_{0}=2.71$, $K / V^{1 / 2}=2.71$; and (b) numerical: $\theta_{0}^{a d v}=0.6, \delta=0.015$; asymptotic (dot-dashed) $\theta_{0}^{a d v}=0.6, \delta=$ $0.019, s_{0}=3.08, K / V^{1 / 2}=1.98$; asymptotic (dashed) $\theta_{0}^{a d v}=0.69, \delta=0.015, s_{0}=4.16, K / V^{1 / 2}=$ 2.21. For comparison the equilibrium profile of a droplet on a homogeneous substrate without wettability gradient is also shown (dotted lines). 
* Electronic address: pismen@ @echunix.technion.ac.il

$\dagger$ Electronic address: thiele@pks.mpg.de, URL: http://www.uwethiele.de

1 C. Huh and L. E. Scriven, "Hydrodynamic model of steady movement of a solid / liquid / fluid contact line,” J. Colloid Interface Sci. 35, 85-101 (1971).

2 H. K. Moffatt, "Viscous and resistive eddies near a sharp corner," J. Fluid Mech. 18, 1-18 (1964).

3 M. A. Spaid and G. M. Homsy, "Stability of Newtonian and viscoelastic dynamic contact lines," Phys. Fluids 8, 460-478 (1996).

4 L. M. Hocking, "A moving fluid interface. II. The removal of the force singularity by a slip flow," J. Fluid Mech. 79, 209-229 (1977).

5 H. P. Greenspan, "On the motion of a small viscous droplet that wets a surface (relevant to cell movement)," J. Fluid Mech. 84, 125-143 (1978).

6 P.-G. de Gennes, “Wetting: Statistics and dynamics," Rev. Mod. Phys. 57, 827-863 (1985).

7 G. F. Teletzke, H. T. Davis, and L. E. Scriven, “Wetting hydrodynamics,” Rev. Phys. Appl. 23, 989-1007 (1988).

8 E. B. Dussan, "On the spreading of liquids on solid surfaces: Static and dynamic contact lines," Ann. Rev. Fluid Mech. 11, 371-400 (1979).

9 P. Seppecher, “Moving contact lines in the Cahn-Hilliard theory,” Int. J. Eng. Sci. 34, 977-992 (1996).

10 Y. D. Shikhmurzaev, "Moving contact lines in liquid/liquid/solid systems," J. Fluid Mech. 334, 211-249 (1997).

11 L. M. Hocking and M. J. Miksis, "Stability of a ridge of fluid," J. Fluid Mech. 247, 157-177 (1993).

12 D. E. Kataoka and S. M. Troian, "A theoretical study of instabilities at the advancing front of thermally driven coating films," J. Colloid Interface Sci. 192, 350-362 (1997).

13 A. L. Bertozzi and M. P. Brenner, "Linear stability and transient growth in driven contact lines," Phys. Fluids 9, 530-539 (1997).

14 B. V. Derjaguin, N. V. Churaev, and V. M. Muller, Surface Forces, Consultants Bureau, New York (1987).

15 J. N. Israelachvili, Intermolecular and Surface Forces, Academic Press, London (1992).

${ }^{16}$ L. M. Pismen, "Nonlocal diffuse interface theory of thin films and the moving contact line," Phys. Rev. E 6402, 021603 (2001).

17 A. Oron, S. H. Davis, and S. G. Bankoff, "Long-scale evolution of thin liquid films," Rev. Mod. Phys. 
69, 931-980 (1997).

18 V. S. Mitlin, "Dewetting of solid surface: Analogy with spinodal decomposition," J. Colloid Interface Sci. 156, 491-497 (1993).

19 A. Sharma and R. Khanna, "Pattern formation in unstable thin liquid films," Phys. Rev. Lett. 81, 34633466 (1998).

20 A. Oron, "Three-dimensional nonlinear dynamics of thin liquid films," Phys. Rev. Lett. 85, 2108-2111 (2000).

21 A. L. Bertozzi, G. Grün, and T. P. Witelski, "Dewetting films: Bifurcations and concentrations," Nonlinearity 14, 1569-1592 (2001).

22 U. Thiele, M. G. Velarde, and K. Neuffer, "Dewetting: Film rupture by nucleation in the spinodal regime,” Phys. Rev. Lett. 87, 016104 (2001).

23 J. Becker, G. Grün, R. Seemann, H. Mantz, K. Jacobs, K. R. Mecke, and R. Blossey, "Complex dewetting scenarios captured by thin-film models," Nature Mat. 2, 59-63 (2003).

${ }^{24}$ M. H. Eres, L. W. Schwartz, and R. V. Roy, "Fingering phenomena for driven coating films," Phys. Fluids 12, 1278-1295 (2000).

25 M. Bestehorn and K. Neuffer, "Surface patterns of laterally extended thin liquid films in three dimensions,” Phys. Rev. Lett. 87, 046101,1-4 (2001).

26 U. Thiele and E. Knobloch, "Front and back instability of a liquid film on a slightly inclined plate," Phys. Fluids 15, 892-907 (2003).

27 J. Eggers, "Toward a description of contact line motion at higher capillary numbers," Phys. Fluids 16, 3491-3494 (2004).

28 J. Eggers, "Hydrodynamic theory of forced dewetting," Phys. Rev. Lett. 93, 094502 (2004).

29 J. Eggers, "Existence of a moving contact line," Phys. Fluids (2005), (in press).

30 C. M. Bender and S. A. Orszag, Advanced mathematical methods for scientists and engineers, Mc GrawHill, New York (1978).

31 W. F. Ford, “A third-order differential equation,” SIAM Rev. 34, 121-122 (1992).

32 B. R. Duffy and S. K. Wilson, "A third-order differential equation arising in thin-film flows and relevant to Tanner's law," Appl. Math. Lett. 10, 63-68 (1997).

33 O. V. Voinov, "Slow wetting of a solid by a liquid film from a moving meniscus," J. Colloid Interface Sci. 188, 1-8 (1997).

34 E. Raphaël, “Spreading of droplets on a patchy surface," C. R. Acad. Sci. Ser. II 306, 751-754 (1988). 
35 F. Brochard, "Motions of droplets on solid-surfaces induced by chemical or thermal-gradients," Langmuir 5, 432-438 (1989).

36 S. Daniel and M. K. Chaudhury, "Rectified motion of liquid drops on gradient surfaces induced by vibration," Langmuir 18, 3404-3407 (2002).

37 R. J. Petrie, T. Bailey, C. B. Gorman, and J. Genzer, "Fast directed motion of "fakir" droplets," Langmuir 20, 9893-9896 (2004).

38 S. Daniel, S. Sircar, J. Gliem, and M. K. Chaudhury, "Ratcheting motion of liquid drops on gradient surfaces," Langmuir 20, 4085-4092 (2004).

39 F. Domingues Dos Santos and T. Ondarçuhu, "Free-running droplets," Phys. Rev. Lett. 75, 2972-2975 (1995).

40 S. W. Lee, D. Y. Kwok, and P. E. Laibinis, "Chemical influences on adsorption-mediated self-propelled drop movement," Phys. Rev. E 65, 051602 (2002).

41 U. Thiele, K. John, and M. Bär, "Dynamical model for chemically driven running droplets," Phys. Rev. Lett. 93, 027802 (2004).

42 K. John, M. Bär, and U. Thiele, "Self-propelled running droplets on solid substrates driven by chemical reactions,” Eur. Phys. J. E (2005), (in press).

43 A. Yochelis and L. M. Pismen, "Droplet motion driven by surface freezing or melting: A mesoscopic hydrodynamic approach,” Phys. Rev. E 72, 025301(R) (2005).

44 A. Sharma, "Relationship of thin film stability and morphology to macroscopic parameters of wetting in the apolar and polar systems," Langmuir 9, 861-869 (1993).

45 E. Doedel, H. B. Keller, and J. P. Kernevez, "Numerical analysis and control of bifurcation problems (I) Bifurcation in finite dimensions," Int. J. Bif. Chaos 1, 493-520 (1991).

46 E. Doedel, H. B. Keller, and J. P. Kernevez, "Numerical analysis and control of bifurcation problems (II) Bifurcation in infinite dimensions,” Int. J. Bif. Chaos 1, 745-72 (1991).

47 E. J. Doedel, A. R. Champneys, T. F. Fairgrieve, Y. A. Kuznetsov, B. Sandstede, and X. J. Wang, AUTO97: Continuation and bifurcation software for ordinary differential equations, Concordia University, Montreal (1997).

48 L. M. Pismen and Y. Pomeau, "Mobility and interactions of weakly nonwetting droplets," Phys. Fluids 16, 2604-2612 (2004).

49 U. Thiele, M. G. Velarde, K. Neuffer, M. Bestehorn, and Y. Pomeau, "Sliding drops in the diffuse interface model coupled to hydrodynamics,” Phys. Rev. E 64, 061601 (2001). 
50 U. Thiele, K. Neuffer, M. Bestehorn, Y. Pomeau, and M. G. Velarde, "Sliding drops on an inclined plane," Colloid Surf. A - Physicochem. Eng. Asp. 206, 87-104 (2002).

51 U. Thiele and E. Knobloch, “Thin liquid films on a slightly inclined heated plate," Physica D 190, 213 248 (2004). 